\title{
A OBESIDADE COMO FATOR PREDITIVO DE HOSPITALIZAÇÃO EM UTI NO PACIENTE ADULTO INFECTADO COM COVID-rg: UMA REVISÃO INTEGRATIVA
}

\author{
OBESITY AS A PREDICTIVE FACTOR FOR THE INTENSIVE CARE UNIT \\ HOSPITALIZATION IN INTECTED ADULT PATIENTS WITH COVID-I9: AN \\ INTEGRATIVE REVIEW
}

\section{Diomar Maria do Nascimento Araújo' ${ }^{1}$ Islane Cristina Martins ${ }^{2}$}

RESUMO: Introdução: A obesidade parece ter forte relação com as complicações da COVID-ı. Não só isso, mas na literatura existente relaciona-se a obesidade a desfechos ruins. Desse modo, indivíduos obesos são propensos às vias aéreas diminuídas, tendo o potencial respiratório afetado. Objetivo: Realizar uma revisão integrativa da literatura a fim de avaliar a obesidade e outros potenciais fatores preditivos para o agravamento da COVID-i9 em pacientes adultos hospitalizados. Material e Métodos: Foi feito um levantamento da literatura em agosto de 202I, nas bases de dados dos Periódicos CAPES, Google Scholar e Pubmed. Os descritores utilizados foram os seguintes: "Fatores de Risco" AND "Infecções por Coronavirus" AND " Obesidade" AND "Unidades de Terapia Intensiva" e, em inglês, "Risk Factors" AND " Coronavirus Infections" AND " Obesity" AND " Intensive Care Units" em todas as bases de dados. Foram selecionados ro artigos sendo incluídos segundo os critérios de elegibilidade. Resultados: Como resultados, foi possível observar que $40 \%$ apresentaram prevalência do gênero masculino na necessidade aumentada de ventilação mecânica invasiva (VMI) e internação na unidade terapia intensiva (UTI), 50\% dos estudos associaram a idade avançada ao principal fator de risco á COVID-ı́ e a maior mortalidade, dois estudos $20 \%$ relacionaram a etnia negra e parda como fator de risco predominante, cinco estudos 50\% associaram a comorbidade como fator agravante do novo Coronavírus, cinco estudos $50 \%$ correlacionaram o sobrepeso e a obesidade ao aumento da admissão em UTI, a necessidade de VMI e risco maior de morte, sendo os pacientes com o IMC entre $30 \mathrm{e}$ $35 \mathrm{~kg} / \mathrm{m} 2$ os mais críticos. Conclusão: Os achados desta revisão permitiram concluir que o sobrepeso e a obesidade foram fatores preditivos para agravamento da COVIDI9 em pacientes hospitalizados. Bem como, o gênero masculino, a idade avançada e a etnia negra e parda.

\footnotetext{
I Especialista em Saúde Pública com ênfase em Saúde da Família- Sanitarista pela Faculdade José Lacerda Filho Ciências Aplicadas-FAJOLCA. Email: diomarnutri@hotmail.com.

${ }^{2}$ Doutora em Neurociências pela Universidade Federal de Pernambuco-UFPE.
} 
Palavras-chave: Fatores de Risco. Infecções por Coronavírus. Obesidade. Unidades de Terapia Intensiva.

ABSTRACT: Introduction: The obesity seems to have an intense relationship with the complications of COVID-I9. Not just this, but in the existing literature, obesity is related to poor outcomes. Thus, non-obese people are prone to reduced airways, and their respiratory potential is affected. Objective: Carrying out an integrative literature review in order to assess obesity and other potential predictive factors for the worsening of COVID-I9 in hospitalized adult patients. Materials e Methods: It was carried out an literature survey in August 2021, in the CAPES, Google Scholar and PubMed journals databases. It was used the following descriptors: "Risk Factors" AND " Coronavirus Infections" AND " Obesity" AND " Intensive Care Units" and, in English, "Risk Factors" AND " Coronavirus Infections" AND " Obesity" AND " Intensive Care Units" in all databases. It was selected ten articles and included according to the eligibility criteria. Results: As a result, it was noted that $40 \%$ showed a prevalence of males in the increased need for invasive mechanical ventilation (IMV) and admission to the intensive care unit (ICU), 50\% of the studies associated advanced age to the main risk factor to COVID- 19 and higher mortality, two studies $20 \%$ related black and mixed race as a predominant risk factor, five studies $50 \%$ associated comorbidity as an aggravating factor of the new Coronavirus, five studies $50 \%$ correlated overweight and obesity with increased admission in ICU, the need for IMV and increased risk of death, with patients with a BMI between 30 and $35 \mathrm{~kg} / \mathrm{m} 2$ being the most critical. Conclusion: The findings of this review permitted us to conclude that overweight and obesity were predictive factors for the worsening of COVID-I9 in hospitalized patients. As well as, male gender, advanced age and black and mixed ethnicity.

Key-words: Risk factors. Coronavirus Infections. Obesity. Intensive Care Units.

\section{INTRODUÇÃO}

No mês de dezembro do ano de 2019 surgiram casos de pneumonia na China, identificada na cidade de Wuhan, como o novo coronavírus, nomeada de Síndrome Respiratória Aguda Grave - Coronavírus 2 (SARS-CoV-2) (McMICHAEL et al.,2020).

Logo, os sintomas mais frequentes na admissão, foram febre, tosse, expectoração e fadiga (ZHOU et al.,2020). Sendo assim, são fatores agravantes da síndrome respiratória, os pacientes do sexo masculino, idade acima dos 65 anos e em alguns estudos a etnia (SILVA, I. et al.,202I). 
Dito isso, a complicação constantemente observada foi sepse, seguida por insuficiência respiratória, síndrome do desconforto respiratório agudo (SDRA), insuficiência cardíaca e choque séptico (ZHOU et al.,2020).

Portanto, a principal complicação pulmonar do coronavírus é a pneumonia, que pode evoluir para a forma fatal. Além disso, seus sintomas também são sugestivos de COVID-I9, como a dispneia e principalmente os infiltrados bilaterais com opacidade em vidro fosco analisados em exames de imagem (ZAIM et al., 2020).

Nesse sentido, as evidências associam fortemente a obesidade a fatores de risco para COVID-19. Dessa forma, indivíduos com comorbidades preexistentes como a diabetes mellitus, hipertensão e obesidade grave são mais predispostos a ser infectado (BANERJEE et al., 2020).

Sendo assim, parece haver forte relação entre a obesidade e as complicações da COVID-19. Não só isso, mas a literatura relaciona a obesidade a desfechos ruins. Desse modo, obesos são propensos às vias aéreas diminuídas, tendo o potencial respiratório afetado (SILVA, G. et al.,202I).

E, uma das formas mundialmente adotada para avaliar o estado nutricional é através da mensuração do Índice de Massa Corporal (IMC). Para calcular esse índice divide-se o peso do indivíduo pela sua altura elevada ao quadrado $(\mathrm{kg} / \mathrm{m} 2)$ (ARROYO-JOHNSON; MINCEY, 2016).

Então, a classificação de sobrepeso e pré-obesidade, quando o ponto de corte estiver entre 25,0 e $29,9 \mathrm{~kg} / \mathrm{m} 2$ e para ser definido como obeso, o IMC deve ser igual ou estar acima de $30 \mathrm{~kg} / \mathrm{m} 2$. E, quanto à normalidade é considerada quando estiver entre 18,5 e 24,9 kg/m2 (ARROYO-JOHNSON; MINCEY, 20I6).

Dessa maneira, a obesidade grave (IMC $\geq 35 \mathrm{~kg} / \mathrm{m} 2)$ foi associada à admissão na UTI, enquanto história de doença cardíaca e obesidade (IMC $\geq 30 \mathrm{~kg} / \mathrm{m} 2$ ) foram independentemente associadas ao uso de ventilação mecânica invasiva (VMI) (KALLIGEROS et al.,2020).

Com isso, a obesidade aumenta a suscetibilidade e a mortalidade por COVIDI9, através da SARS-CoV que se liga as células- alvo pelo receptor da enzima 
conversora de angiotensina 2 (ECA-2) (SILVA, G. et al.,202I), pela ativação do sistema renina-angiotensina (SRA) (LI et al., 2003).

Dessa forma, a ECA2 é o receptor celular para o vírus SARS-CoV-2, na COVID-ı, porque o vírus contém uma proteína de membrana chamada Spike, que se liga à ECA2 de forma facilitada, replicando e causando desregulação (COSTA et al., 2020).

Diante do exposto, a obesidade tem sido um fator de risco para diversos agravos, como mortalidade por COVID-ı9 e aumento das hospitalizações (SIMONNET et al., 2020).

Logo, essa afecção representa um grande desafio ao setor da saúde. Assim, é imperativo um estudo que amplie o conhecimento sobre a temática e avalie esses dois problemas de saúde pública mundial, a infecção pelo novo coronavírus e a obesidade. Conduzindo futuras pesquisas na área.

Portanto, o objetivo dessa revisão integrativa foi avaliar a obesidade e outros potenciais fatores preditivos para o agravamento da COVID-I9 em pacientes adultos hospitalizados.

\section{MATERIAL E MÉTODOS}

O levantamento da literatura foi realizado em junho de 2021, nas bases de dados Periódicos CAPES, Google Scholar e Pubmed. Os descritores utilizados foram os seguintes: "Fatores de risco" AND "Infecções por coronavirus" AND "Obesidade" AND 'Unidades de Terapia Intensiva" e, em inglês, "Risk Factors" AND "Coronavirus infections" AND 'Obesity" AND "Intensive Care Units" em todas as bases de dados. Foram selecionados io artigos sendo incluídos segundo os critérios de elegibilidade conforme a Figura I. Os critérios de inclusão foram: artigos nos idiomas inglês e português, publicados durante o período de 2019 a 2021, priorizaram-se artigos que avaliaram a obesidade e outros potenciais fatores preditivos para o agravamento da COVID-I9 em pacientes adultos hospitalizados no centro de terapia intensiva. Os critérios de exclusão foram artigos de revisão de literatura ou metanálise. 
FIGURA I. FLUXOGRAMA E CRITÉRIOS DE SELEÇÃO E INCLUSÃO DOS TRABALHOS

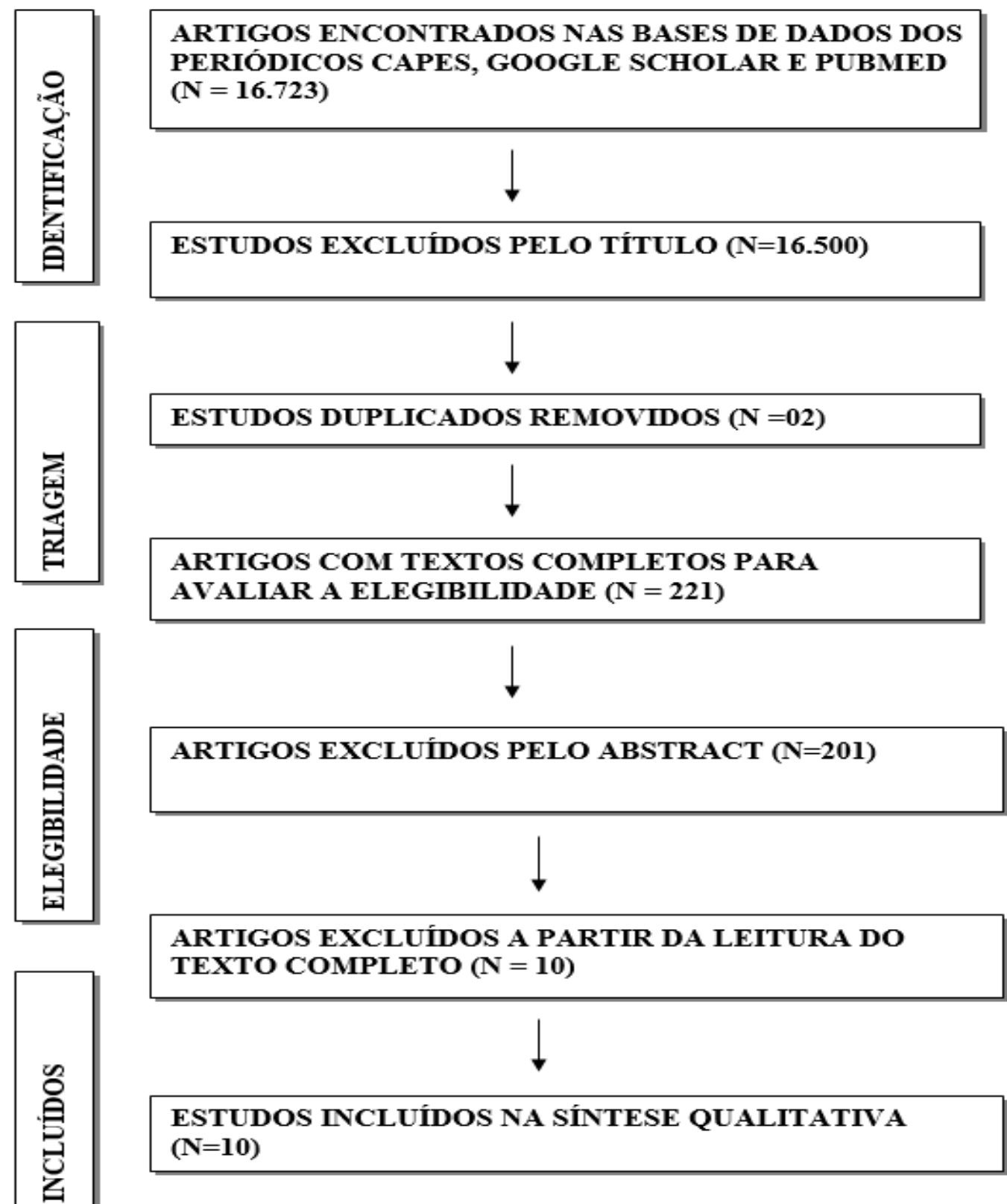

2 RESULTADOS

Os resultados do presente estudo encontram-se na Tabela I. 
Revista Ibero- Americana de Humanidades, Ciências e Educação- REASE open $\odot$ Access

Tabela I - Demonstrativo dos artigos que integram a Revisão Integrativa

\begin{tabular}{|c|c|c|c|c|c|c|}
\hline$\# \mathbf{N}$ & Data & Título & Autores & Periódico & Objetivos & Resultados \\
\hline I & 2021 & $\begin{array}{l}\text { Risk factors for } \\
\text { critical illness } \\
\text { and death } \\
\text { among adult } \\
\text { Brazilians with } \\
\text { COVID-ı9 }\end{array}$ & $\begin{array}{l}\text { Isabela Silva, Natália Cristina de Faria, Álida } \\
\text { Rosária Silva Ferreira, Lucilene Rezende Anastácio } \\
\text { and Lívia Garcia Ferreira }\end{array}$ & $\begin{array}{l}\text { Rev Soc Bras } \\
\text { Med Trop }\end{array}$ & $\begin{array}{l}\text { Verificar e analisar o impacto } \\
\text { de variáveis demográficas e } \\
\text { comórbidas como fatores de } \\
\text { risco para admissão em UTI e } \\
\text { mortalidade na população } \\
\text { adulta brasileira. }\end{array}$ & 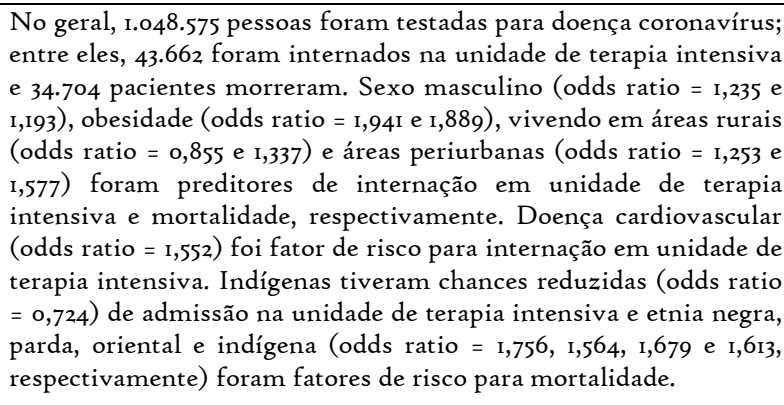 \\
\hline 2 & 2021 & $\begin{array}{l}\text { Perfil } \\
\text { epidemiológico e } \\
\text { preditores de } \\
\text { fatores de risco } \\
\text { para a COVID- } \\
\text { I9 na região sul } \\
\text { do Brasil }\end{array}$ & $\begin{array}{l}\text { Sarah Gisele Martins Klokner, Ramsés Antunes da } \\
\text { Luz, Pedro Henrique de Moura Araujo, Janete } \\
\text { Knapik, Synara Sepúlveda Sales, Grasiela Torrico, } \\
\text { Fernanda Pereira Labiak, Maria Julia Pegoraro Gai } \\
\text {, Clarissa Venturieri, Rafael Frasson, Adelino } \\
\text { Domingos Onofre e Roberto Moraes Cruz }\end{array}$ & $\begin{array}{l}\text { Research, } \\
\text { Society and } \\
\text { Development }\end{array}$ & $\begin{array}{l}\text { Analisar o } r \text { perfil } \\
\text { epidemiológico, bem como } \\
\text { analisar as variáveis } \\
\text { preditivas de risco e proteção } \\
\text { para COVID-Ig, na região } \\
\text { Sul do Brasil. }\end{array}$ & $\begin{array}{l}\text { Indicaram que as variáveis estado nutricional, sexo, idade e } \\
\text { comorbidade predizem a condição de pessoas infectadas se } \\
\text { recuperarem, morrerem, manifestarem sintomas ou serem } \\
\text { hospitalizadas por COVID-Ig. }\end{array}$ \\
\hline 3 & 2021 & $\begin{array}{l}\text { Risk Factors } \\
\text { Associated } \\
\text { With Mortality } \\
\text { Among Patients } \\
\text { With COVID- } \\
\text { I9 in Intensive } \\
\text { Care Units in } \\
\text { Lombardy, Italy }\end{array}$ & $\begin{array}{l}\text { GiacomoGrasselli,Massimiliano } \\
\text { Greco,AlbertoZanella,Giovanni Albano, Massimo } \\
\text { Antonelli, Giacomo Bellani, Ezio Bonanomi,Luca } \\
\text { Cabrini, Eleonora Carlesso, Gianpaolo } \\
\text { Castelli,Sergio Catraneo,Danilo } \\
\text { Cereda,SergioColombo,Antonio } \\
\text { Coluccello,Giuseppe Crescini, Andrea Forastieri } \\
\text { Molinari, Giuseppe Foti,Roberto Fumagalli,Giorgio } \\
\text { Antonior Iotti,Thomas Langer,Nicola } \\
\text { Latronico,Ferdinando Luca Lorini, Francesco } \\
\text { Mojoli,Giuseppe Natalini, Carla Maria Pessina, } \\
\text { Vito Marco Ranieri,Roberto Rech, Luigia } \\
\text { Scudeller,Antonio Rosano, Enrico Storti, B. Taylor }\end{array}$ & $\begin{array}{l}\text { JAMA } \\
\text { Internal } \\
\text { Medicine. }\end{array}$ & $\begin{array}{l}\text { Avaliar os fatores de risco } \\
\text { independentes associados à } \\
\text { mortalidade de pacientes com } \\
\text { COVID-19 em UTI na } \\
\text { Lombardia, Itália. }\end{array}$ & $\begin{array}{l}3988 \text { pacientes. Idade média de } 63 \text { anos; } 3188 \text { eram homens, e } 1998 \text { de } \\
3300 \text { tinha pelo menos I comorbidade. Admissão na UTI, } 2.929 \\
\text { necessitou de VMI. Acompanhamento médio foi de } 44 \text { dias; o } \\
\text { tempo médio desde o início dos sintomas até UTI foi de Io dias; o } \\
\text { tempo médio de permanência na UTI foi de I2 dias; As taxas de } \\
\text { mortalidade hospitalar e na UTI foram I2 e } 27 \text { por Iooo pacientes- } \\
\text { dia2 Bespectivamente. Dos primeiros I. } 715 \text { pacientes, em maio de } \\
2020,50,4 \% \text { tiveram alta da UTI, } 48,7 \% \text { morreram na UTI e o,8\% } \\
\text { ainda estavam na UTI; no geral, pacientes } 53,4 \% \text { morreram no } \\
\text { hospital. Fatores de risco independentes associados com } \\
\text { mortalidade incluída idade avançada, sexo masculino, alta fração de } \\
\text { oxigênio inspirado, pressão expiratória final positiva alta. } \\
\text { Admissão na UTI e história de DPOC, hipercolesterolemia e }\end{array}$ \\
\hline
\end{tabular}


Revista Ibero- Americana de Humanidades, Ciências e Educação- REASE $\quad$ open $\mathbf{O}$ Access

\begin{tabular}{|c|c|c|c|c|c|c|}
\hline$\# \mathbf{N}$ & Data & Título & Autores & Periódico & Objetivos & Resultados \\
\hline & & & $\begin{array}{l}\text { Thompson, Marcello Tirani, Pier Giorgio Villani, } \\
\text { Antonio Pesenti, Maurizio Cecconi. }\end{array}$ & & & $\begin{array}{l}\text { diabetes tipo } 2 \text {. Nenhuma medicação foi independentemente } \\
\text { associada à mortalidade. }\end{array}$ \\
\hline \multicolumn{7}{|c|}{ Continua } \\
\hline 4 & 2020 & $\begin{array}{l}\text { Aspectos } \\
\text { demográficos e } \\
\text { socioeconômicos } \\
\text { dos adultos } \\
\text { brasileiros e a } \\
\text { COVID-I9: uma } \\
\text { análise dos } \\
\text { grupos de risco a } \\
\text { partir da } \\
\text { Pesquisa } \\
\text { Nacional de } \\
\text { Saúde, 2013 }\end{array}$ & Gabriel Mendes Borges e Claudio Dutra Crespo & $\begin{array}{l}\text { Cad. Saúde } \\
\text { Pública }\end{array}$ & $\begin{array}{l}\text { Caracterizar os grupos de } \\
\text { risco para COVID-19 no } \\
\text { Brasil, bem como estimar o } \\
\text { número de indivíduos } \\
\text { convivendo no mesmo } \\
\text { domicílio com pessoas no } \\
\text { grupo de risco. }\end{array}$ & $\begin{array}{l}\text { Mostram que a idade é o principal fator de risco para comorbidades } \\
\text { associadas à COVID-19, mas há também maior risco para os } \\
\text { vulneráveis, menos escolarizados e pretos e pardos. Estima-se que } \\
68,7 \% \text { dos brasileiros viviam com pelo menos uma pessoa no grupo } \\
\text { de risco,30,3\% viviam com pelo menos um idoso e } 38,4 \% \text { não tinham } \\
\text { idosos no domicílios, mas havia pelo menos um adulto com } \\
\text { condiçóes médicas preexistentes. A proporção de pessoas vivendo } \\
\text { com pelo menos um morador no grupo de risco era maior ou igual a } \\
\text { 5o\% para todas as idades, sendo crescente a partir dos } 35 \text { anos, } \\
\text { também um alto número com idades entre ro e } 25 \text { anos convivendo } \\
\text { com pessoas no grupo de risco. }\end{array}$ \\
\hline
\end{tabular}

Um total de 92 pacientes (6r,9\% homens; idade $70,5[13,3]$ anos) foram inscritos. Os pacientes com sobrepeso e obesidade eram mais jovens do que os pacientes com peso normal (68,o $[12,6]$ e $67, \mathrm{o}[12,6]$ anos vs. 76,I $[13,0]$ anos, $\mathrm{P}<\mathrm{o}, \mathrm{oI})$. Uma maior necessidade de

Avaliar a relação entre a gravidade do COVID-ig e a classificação da obesidade de acordo como o IMC.
Association of

Disease Severity

Among Patients

with

Coronavirus
Markos Kalligeros, Fadi Shehadeh, Evangelia K. Mylonar, Gregorio Benitez, Curt G. Beckwithı, Obesity

Philip A. Chanı, e Eleftherios Mylonakis ventilação assistida além do suporte de oxigênio puro (ventilação mênica invasiva ou ventilas̆o ño invasiva) e uma maior admissão unvidades de terapia intoniva ou semi-intensiva foram observadas em pacientes com sobrepeso e obesidade ( $\mathrm{P}<0$,or e $\mathrm{P}$ $<0,05$, respectivamente) mesmo após o ajuste para sexo, idade comorbidades ( $\mathrm{P}<0,05$ e $\mathrm{P}<\mathrm{o}, \mathrm{ooI}$, respectivamente) ou quando os pacientes com demência ou câncer avançado foram removidos $d$ análise $(\mathrm{P}<0,05)$.

Explorar a associação De236e fevereiro a 5 de abril, ro3 pacientes consecutivos foram potencial de obesidade e hospitalizados com COVID-19. Destes, 44 pacientes $(42,7 \%)$ foram outras doenças crônicas com admitidos em UTI e $29(65,9 \%)$ necessitaram de VMI. A desfechos graves, como prevalência de obesidade foi de 47,5\% (49 de ro3). Em uma análise admissão em unidade de multivariada, a obesidade grave $(\mathrm{IMC} \geq 35 \mathrm{~kg} / \mathrm{m} 2)$ foi associada à terapia intensiva (UTI) e admissão na UTI (odds ratio ajustada [aOR]: 5,39, IC 95\%: I,13ventilação mecânica invasiva 25,64). Além disso, os pacientes que necessitaram de IMV eram (VMI), em pacientes mais propensos a ter doença cardíaca (aOR: 3,4I, IC de 95\%: I,05- 
Revista Ibero- Americana de Humanidades, Ciências e Educação- REASE $\quad$ open Access $^{\text {acto }}$

\begin{tabular}{|c|c|c|c|c|c|c|}
\hline$\# N$ & Data & Título & Autores & Periódico & Objetivos & Resultados \\
\hline & & & & & $\begin{array}{l}\text { hospitalizados com doença } \\
\text { coronavírus 2019 (COVID- } \\
\text { 19). }\end{array}$ & $\begin{array}{l}\text { II,06), obesidade (IMC = 30-34,9 kg / m2; aOR: 6,85, IC de 95\%: 1,05- } \\
\text { 44,82), ou obesidade grave (IMC } \geq 35 \mathrm{~kg} / \mathrm{m2} \text {; aOR: 9,99, IC de 95\%: } \\
\text { I,39-71,69). }\end{array}$ \\
\hline 7 & 2020 & $\begin{array}{l}\text { How important } \\
\text { is obesity as a } \\
\text { risk factor for } \\
\text { respiratory } \\
\text { failure, } \\
\text { intensive care } \\
\text { admission and } \\
\text { death in } \\
\text { hospitalised } \\
\text { COVID-rg } \\
\text { patients? Results } \\
\text { from a single } \\
\text { Italian centre }\end{array}$ & 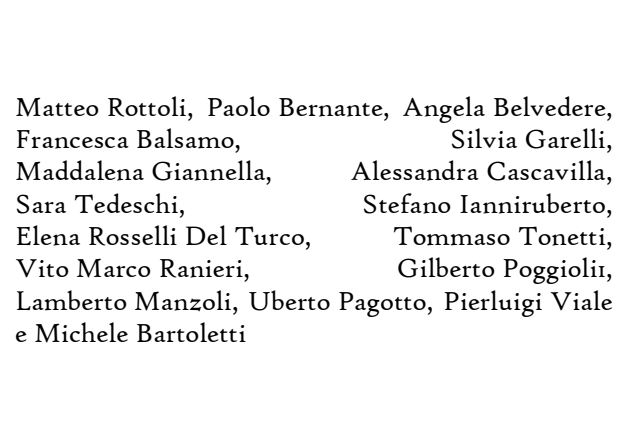 & $\begin{array}{l}\text { European } \\
\text { Journal of } \\
\text { Endocrinology }\end{array}$ & $\begin{array}{l}\text { Analisar se o IMC } \\
\text { representava fator de risco } \\
\text { para insuficiência } \\
\text { respiratória, admissão em } \\
\text { unidade de terapia intensiva } \\
\text { (UTI) e óbito. }\end{array}$ & 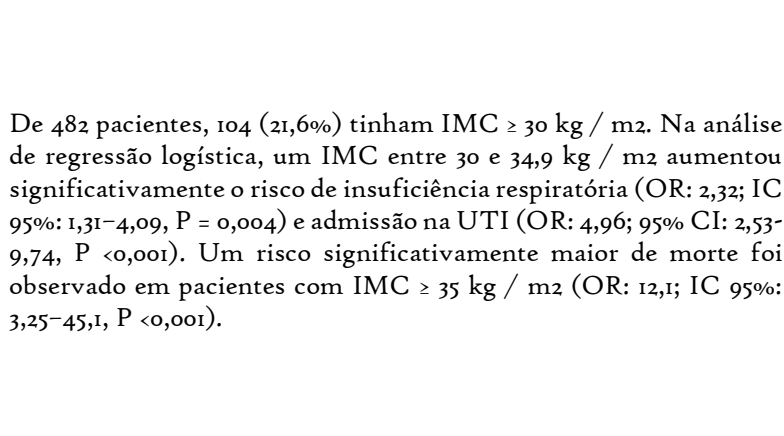 \\
\hline 8 & 2020 & $\begin{array}{l}\text { Clinical course } \\
\text { and risk factors } \\
\text { for mortality of } \\
\text { adult inpatients } \\
\text { with COVID-I9 } \\
\text { in Wuha, } \\
\text { China: a } \\
\text { retrospective } \\
\text { cohort study }\end{array}$ & $\begin{array}{l}\text { Fei Zhou, Ting Yu, Ronghui Du, Guohui Fan, Ying } \\
\text { Liu, Zhibo Liu, Jie Xiang, Yeming Wang, Bin Song, } \\
\text { Xiaoying Gu, Lulu Guan, Yuan Wei, Hui Li, } \\
\text { Xudong Wu, Jiuyang Xu, Shengjin Tu, Yi Zhang, } \\
\text { Hua Chen, Bin Cao }\end{array}$ & The lancet & $\begin{array}{l}\text { Explorar os fatores de risco de } \\
\text { morte intra-hospitalar para os } \\
\text { pacientes e descrever o curso } \\
\text { clínico dos sintomas, } \\
\text { eliminação viral e } \\
\text { laboratterações } \\
\text { hospitalizaçãa. na na }\end{array}$ & 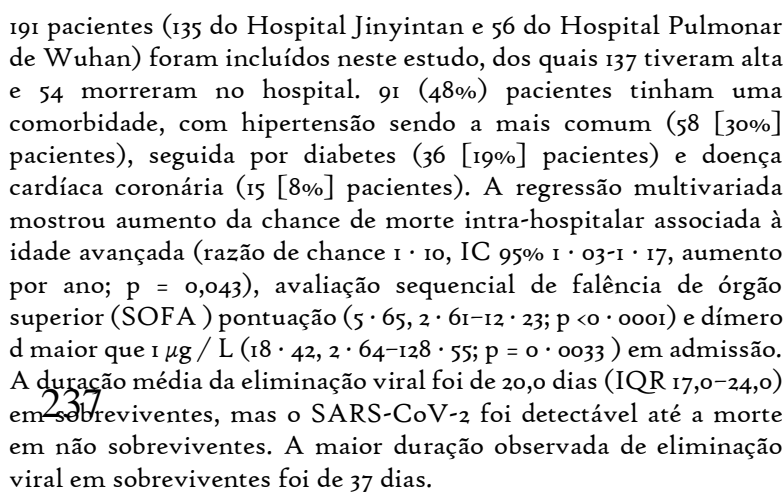 \\
\hline
\end{tabular}

\section{Continua}




\begin{tabular}{|c|c|c|c|c|c|c|c|}
\hline$\# \mathrm{~N}$ & Data & Título & Autores & Periódic & & Objetivos & Resultados \\
\hline 9 & 2020 & $\begin{array}{l}\text { High Prevalence } \\
\text { of Obesity in } \\
\text { Severe Acute } \\
\text { Respiratory } \\
\text { Syndrome } \\
\text { Coronavirus-2 } \\
\text { (SARS-CoV-2) } \\
\text { Requiring } \\
\text { Invasive } \\
\text { Mechanical } \\
\text { Ventilation }\end{array}$ & $\begin{array}{l}\text { Arthur Simonnet, Mikael Chetboun, Julien Poissy, } \\
\text { Violeta Raverdy, Jerome Noulette, Alain Duhamel, } \\
\text { Julien Labreuche, Daniel Mathieu, Francois Pattou, } \\
\text { and Merce Jourdain }\end{array}$ & Obesity & & $\begin{array}{l}\text { Documentar a relação entre } \\
\text { obesidade e e síndrome } \\
\text { respiratória agua grave } \\
\text { coronavírus-2 } \\
\text { 2). }\end{array}$ & 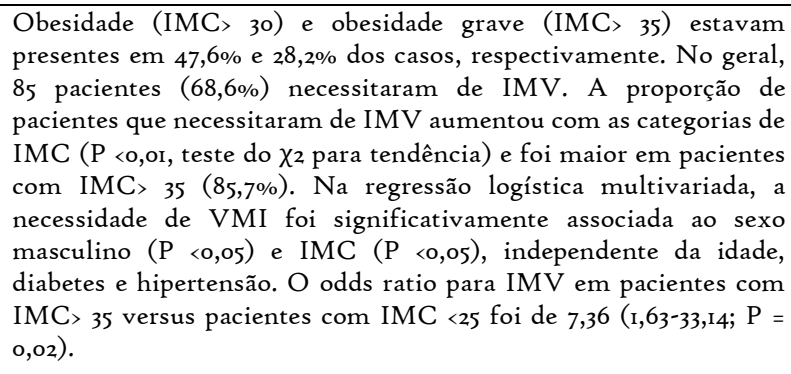 \\
\hline Io & 2020 & $\begin{array}{l}\text { Covid-I9 in the } \\
\text { State of Ceará: } \\
\text { behaviors and } \\
\text { beliefs in the } \\
\text { arrival of the } \\
\text { pandemic }\end{array}$ & $\begin{array}{l}\text { Danilo Lopes Ferreira Lima, Aldo Angelim Dias, } \\
\text { Renata Sabóia Rabelo, gor Demes da Cruz, Samuel } \\
\text { Carvalho Costa, Flávia Maria Noronha Nigri e } \\
\text { Jiovanne Rabelo Neri }\end{array}$ & $\begin{array}{l}\text { Ciência } \\
\text { Saúde } \\
\text { Coletiva }\end{array}$ & & $\begin{array}{l}\text { Avaliar os aspectos } \\
\text { comportamentais e as crenças } \\
\text { da população cearense frente à } \\
\text { pandemia do COVID-Ig. }\end{array}$ & 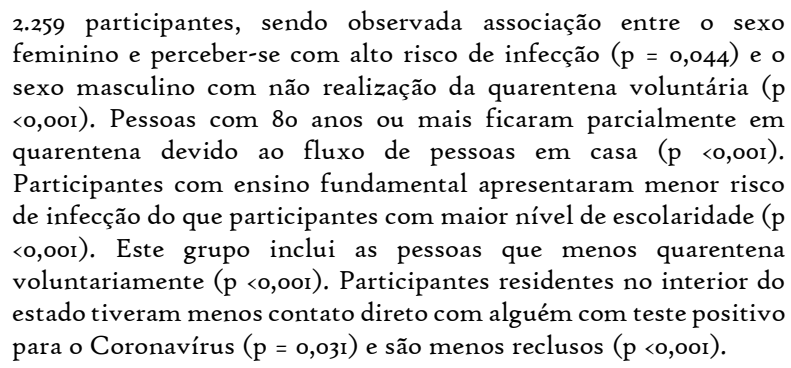 \\
\hline
\end{tabular}




\section{DISCUSSÃO}

O objetivo do presente estudo foi avaliar a obesidade e outros potenciais fatores preditivos para o agravamento da COVID-19 em pacientes adultos internados.

E, de acordo com os artigos pesquisados, a idade foi apontada como fator de risco para prognostico ruim da COVID-ı, no caso os idosos (BORGES; CRESPO, 2020).

Isso se deve ao fato de que essa população demanda ventilação mecânica invasiva (VMI) e geralmente possuem comorbidades (GRASSELLI et al.,202I).

Mas também, ressalta-se que uma idade $\geq 65$ anos foi um forte preditor independente de admissão na unidade de terapia intensiva (UTI) e óbito intrahospitalar (LINDSAY et al., 2020).

Bem como, pessoas com idade entre $75-84$ anos tiveram maior probabilidade de admissão na UTI quando comparado aos de 18-49 anos. E, aqueles com $\geq 85$ anos submeteram-se II vezes mais a mortalidade (LINDSAY et al., 2021).

Nesse sentido, em relação ao gênero, o feminino teve maior taxa de hospitalização, a possível explicação, seria que os homens foram para o hospital já necessitando de cuidados intensivos, além de se exporem mais a situação de risco (SILVA, I. et al,.2021).

Desse modo, os homens foram relacionados ao maior risco de admissão na UTI e morte. Alem disso, outros estudos apontaram que o gênero masculino está associado a maiores taxas de necessidade de VMI (GOYAL et al.,2020).

Além disso, aos grupos étnicos, uma coorte retrospectiva analisou todas as etnias não brancas, aumentaram as taxas de mortalidade devido ao COVID-ı9 (de $56,4 \%$ na raça mista para $75,6 \%$ na população negra). A prevalência dos óbitos era negra e etnias mistas (SILVA, I. et al,.2021).

Isto é, a etnia negra foi mais prevalente, a indígena diminuiu a possibilidade de admissão na UTI por $27,6 \%$. Isso se deve aos indígenas residirem em sua maioria em áreas rurais, distantes de hospitais que possuem UTI, esses fatores aumentando o risco de morte em 24\% 26 (SILVA, I. et al,.202I). 
Dessa forma, às questões sociais no Brasil, em consequência da dificuldade de acesso a saneamento básico e condições de habitação, agrega-se a condição aguda de desigualdade social, com estratos da população vivendo em insegurança (WERNECK; CARVALHO, 2020).

A saber, o estudo de Silva, I. et al., (202I) reafirmar que o nível de educação pode estar relacionado a propagação de doenças infecciosas virais, e também na gênese da obesidade. Sendo assim, um fator de risco para o agravamento do novo coronavírus.

Então, os indivíduos com menor escolaridade e menor renda são mais predispostos a contrair a infecção ao usar o transporte público, além de residirem em lugares aglomerados e com acesso reduzido a assistência médica (LIMA et al.,2020).

Nesse sentido, na admissão ocorreu comumente, linfocitopenia 40\% e 95\% receberam antibióticos e apenas $21 \%$ receberam antivirais. E, o uso de corticosteroide sistemático e imunoterapia intravenosa retardaram os não sobreviventes e sobreviventes (ZHOU et al.,2020).

Além disso, evidenciou-se que os contaminados que não possuíam doenças crônicas não transmissíveis (DCNT), tiveram menor probabilidade de apresentarem sintomas de COVID-ı9, de vir a óbito e de serem hospitalizados, do que os pacientes que tinham DCNT (KLOKNER et al.,202I).

Dito isso, o paciente obeso é mais favorável a ser acometido pelas doenças crônicas não transmissíveis. Essas características expõem essa população a maior letalidade e a um pior prognostico frente ao COVID-I9 (SILVA, R. et al.,202I).

Sendo assim, a hipertensão foi a comorbidade mais prevalente (ZHOU et al.,2020) e os hipertensos tiveram redução no prolongamento da vida, comparado a outras patologias (GRASSELLI et al.,202I).

Bem como, não foi um fator independente relacionado à mortalidade (GRASSELLI et al.,202I). E, apenas 21,6\% do total de pacientes, que não necessitaram de UTI tinha hipertensão (SHAH et al., 2021). 
Desse modo, possivelmente relacionado à ação da enzima conversora de angiotensina (ECA) e dos bloqueadores do receptor de angiotensina (BRA), prescritos no tratamento da hipertensão (DANSER; EPSTEIN; BATLE, 2020).

Isto é, a ECA-2 é expressa no tecido adiposo humano, onde a síndrome respiratória mostra afinidade (MALAVAZOS et al.,2020). Senso assim a ECA-2, receptor que parece facilitar a entrada do coronavírus nas células (DANSER; EPSTEIN; BATLE, 2020).

Portanto, a interação entre ECA-2, Síndrome respiratória Aguda (SRA), tecido adiposo e Coronavírus poderia argumentar o aumento do risco da infecção e dos óbitos. No entanto, o papel da enzima e SRA no COVID-i9 ainda precisam ser esclarecidos (MALAVAZOS et al.,2020).

Além disso, evidencias apontam que paciente sobrepeso tem uma carga viral aumentada e mais tempo de eliminação do vírus, se confrontado aos pacientes não obesos (MAIER et al.,2018).

Nesse sentido, uma coorte retrospectiva estudou obesos em terapia intensiva com a SARS-CoV-2. Sendo, 47,5\% com obesidade com risco moderado (IMC $\geq 30$ ), obesidade grave em 13,7\%, classe II (IMC 35-39,9) e obesidade classe III (IMC $\geq 40$ ) com risco muito grave com $14,5 \%$ (SIMONNET et al.,2020).

Não só isso, mas em um pequeno estudo da França mencionado 124 pacientes com o novo coronavírus, supondo uma relação da necessidade de ventilação mecânica invasiva aumentou com IMC $\geq 35 \mathrm{~kg} / \mathrm{m} 2$ (SHAH et al., 2021).

E, outro estudo em Nova York com 3.615 pacientes, em analogia com indivíduos com IMC <30, onde pacientes com IMC entre 30 e 34,9 tiveram 2,0 e 1,8 vezes mais probabilidade de serem internados em cuidados agudos e críticos, respectivamente (KALLIGEROS et al.,2020).

Além disso, resultados equivalentes foram encontrados por Cai et al., (2020), 47,2\% apresentavam sobrepeso ou obesidade. Bem como, em uma coorte retrospectiva nos Estados Unidos da América (EUA) com 103 pacientes hospitalizados por Coronavírus onde o excesso de peso, foram 47,5\% (49 de I03) (SILVA, G. et al.,2021). 
No entanto, o que se refere à gravidade da doença, a obesidade severa, aumentou o risco de morte e que o risco era maior, independentemente da presença do comorbidades relacionadas á obesidade (ROTTOLI et al.,2020).

Dessa maneira, esses dados alarmantes apontam à longa permanência na UTI, necessidade prolongada de suporte respiratório e alta mortalidade pelo COVID-I9 em pacientes graves (GRASSELLI et al.,2021).

Logo, se faz necessária realização de estudos que correlacione esses dois grandes problemas de saúde mundial, a obesidade e o coranavírus. Em síntese, essas razões apontadas poderiam justificar a obesidade e outros potenciais fatores preditivos como agravante da COVID-I9 em pacientes internados (SILVA, G. et al.,2020).

\section{CONCLUSÃO}

Os achados evidenciam que o sobrepeso e a obesidade foram fatores preditivos para agravamento da COVID-I9 em pacientes hospitalizados. Não só isso, mas pacientes com o Índice de Massa Corpórea (IMC) entre 30 e $35 \mathrm{~kg} / \mathrm{m} 2$ foram os mais críticos.

Bem como, o gênero masculino, a idade avançada e a etnia negra e parda. Desse modo, esses fatores de risco aumentaram a admissão em Unidade de Terapia Intensiva, à necessidade de Ventilação Mecânica Invasiva (VMI) e a mortalidade.

Por isso, ressalta-se que indivíduo obeso infectado pelo vírus ainda é um grande desafio deste século. No entanto, sabe-se que a obesidade pode ser prevenida, que hábitos saudáveis de vida e a perda de peso, podem reduzir a gravidade dessa patologia.

Portanto, diante da pandemia, são necessárias estratégias de combate à obesidade, pois, foi à doença crônica mais grave para todos os desfechos.

\section{REFERÊNCIAS}

ARROYO-JOHNSON, C.; MINCEY, K.D. Obesity Epidemiology Worldwide. Gastroenterol Clin North Am, USA, v.45, n. 4, p. 571-579, 2016. http://dx.doi.org/ro.ıor6/j.gtc.2016.07.or2BANERJEE, M. et al. Obesity and COVID- 
19: A Fatal Alliance. Indian journal of clinical biochemistry. IJCB, vol. 35, n. 4, p. I-8, 2020, https://doi.org/10.1007/s12291-020-00909-2

BORGES, M.G.; CRESPO,D.C. Aspectos demográficos e socioeconômicos dos adultos brasileiros e a COVID-ı: uma análise dos grupos de risco a partir da Pesquisa Nacional de Saúde, 2013. Cadernos de saude publica, Rio de Janeiro, RJ, v. 36, n. io, p. eoor41020, 2020. doi: 10.1590/o102-311Xool41020

CAI, Q. et al., Obesity and COVID-I9 severity in a designated hospital in Shenzhen, Diabetes Care. China. v.43, p.1392-1398, 2020. https://doi.org/I0.2337/dc20-0576

COSTA, B.C.P. et al. Pandemia COVID-ıg e sua relação com a doença cardiovascular: revisão integrativa. Saúde Coletiva, vol. Io, n. 59, p. 4092-4105, 2020.

DANSER, A.H.J; EPSTEIN, M.; BATLE, D. Renin-Angiotensin System Blockers and the COVID-ig Pandemic At Present There Is No Evidence to Abandon ReninAngiotensin System Blockers. Hypertension.2020, 75:00-00. DOI: Io.II6I/HYPERTENSIONAHA.I20.15082.)

GOYAL, P. et al., Clinical characteristics of COVID-ı in New York N Engl J Med, 382:2372-4. 22, 2020, DOI: 10.1056/NEJMc2010419City.

GRASSELLI, G. et al., Risk Factors Associated With Mortality Among Patients With COVID-19 in Intensive Care Units in Lombardy, Italy. JAMA Intern Med. v.18o, n.Io, p.1345-1355, 2021, doi:Io.10oI/jamainternmed.2020.3539.

KALligEROS,M. et al., Association of Obesity with Disease Severity Among Patients with Coronavirus Disease 2019. Obesity, USA, v. 28, n.7,p.1200-1204,2020. doi:10.1002/oby.22859

KLOKNER,S.G.M et al., Perfil epidemiológico e preditores de fatores de risco para a COVID-i9 na região sul do Brasil. Research, Society and Development, Santa Catarina-RN,v. Io, n. 3, p.oI-I3, 202I. DOI: http://dx.doi.org/Io.33448/rsd-vioi3.13197 LI,W et al., Angiotensin-converting enzyme 2 is a functional receptor for the SARS coronavirus. Nature, USA, v.426, p.450-454, 2003. 
LIMA,D.L.F. et al., Covid-ı in the State of Ceará: behaviors and beliefs in the arrival of the pandemic. Cien Saude Colet. v.25, n.5, p.1575-86,2020. https://doi.org/I0.1590/1413-81232020255.07192020

LINDSAY, K. et al., Risk Factors for Intensive Care Unit Admission and In-hospital Mortality Among Hospitalized Adults Identified through the US Coronavirus Disease 2019 (COVID-I9)-Associated Hospitalization Surveillance Network (COVID-NET). Clinical Infectious Diseases, Atlanta, v.72,n. 9,e206-14,2020, DOI: I0.1093/cid/ciaaioı2

MAIER, E.H. et al., Obesity increases the duration of influenza A virus shedding in adults. Journal of Infectious Diseases,v.218,p.1378-1382,2018. https://doi.org/10.1093/ infdis/jiy37o

MALAVAZOS, A.E. et al. Targeting the adipose tissue in COVID-I9. Obesity (Silver Spring, Md.), v. 28, n. 7, p. I178-I179, May 2020. doi:10.1002/oby.22844

MCMICHAEL,T.M. et al. Epidemiology of Covid-19 in a Long-Term Care Facility in King County, Washington. N Engl J Med. v.382,n. 21,p.2005-201I, 2020. DOI: 10.1056/NEJMoa2005412

ROTTOLI, M. et al., How important is obesity as a risk factor for respiratory failure, intensive care admission and death in hospitalised COVID-i9 patients? Results from a single Italian centre. European Journal of Endocrinology. v.183, p.389-39, 2020. https://doi.org/ro.1530/EJE-20-054I

SHAH, H. et al., The triumvirate: why hypertension, obesity, and diabetes are risk factors for adverse efects in patients with COVID-I. Acta Diabetologica, , USA, v. 58,p.831-843,2021. https://doi.org/ro.1007/s00592-020-01636-z

SILVA, G. M. et al., Obesidade como fator agravante da COVID-i9 em adultos hospitalizados: revisão integrativa. Acta Paul Enferm. Paraná, 2021; 34: eAPEo2321. DOI http://dx.doi.org/ro.37689/actaape/202IARo232I

SILVA, I. et al., Risk factors for critical illness and death among adult Brazilians with COVID-I9. Rev Soc Bras Med Trop. Belo Horizonte, MG, v.54, (eoor4-2021), 2021. https://doi.org/10.1590/0037-8682-0014-2021 
SILVA, R. B. et al., Por que a obesidade é um fator agravante para a COVID-I9? Brazilian Journal of Health Review, Curitiba, v.4, n.2, p. 6502-6517 mar./apr. 2021 DOI:I0.34119/bjhrv4n2-20o.

SIMONNET, A. et al. High prevalence of obesity in severe acute respiratory syndrome coronavirus-2 (SARS-CoV-2) requiring invasive mechanical ventilation. Obesity, v. 28, n. 7, p. II95-I199, 2020, doi:10.1002/oby.22831

WERNECK, G. L.; CARVALHO, M. S. A pandemia de Covid-ı9 no Brasil: crônica de uma crise sanitária anunciada. Cadernos de Saúde Pública, v. 36, n. 5, eooo6882o, 2020 .

ZAIM, S. et al. COVID-i9 and Multiorgan Response. Curr Probl Cardiol, vol. 45, n. 8, 100618, 2020, https://doi.org/ı.1016/j.cpcardiol.2020.100618

ZHOU, F. et al. Clinical course and risk factors for mortality of adult inpatients with COVID-I9 in Wuhan, China: a retrospective cohort study. The lancet. China, 2020, doi:10.1016/Sor40-6736(20)30566-3 